Meta

Journal des tradlucteurs

Translators' Journal

\title{
La recherche à l'ESIT : rétrospective et perspectives
}

\section{Colette Laplace}

Volume 40, numéro 1, mars 1995

URI : https://id.erudit.org/iderudit/003397ar

DOI : https://doi.org/10.7202/003397ar

Aller au sommaire du numéro

Éditeur(s)

Les Presses de l'Université de Montréal

ISSN

0026-0452 (imprimé)

1492-1421 (numérique)

Découvrir la revue

Citer cette note

Laplace, C. (1995). La recherche à l'ESIT : rétrospective et perspectives. Meta, 40(1), 162-169. https://doi.org/10.7202/003397ar d'utilisation que vous pouvez consulter en ligne.

https://apropos.erudit.org/fr/usagers/politique-dutilisation/ 
puis de M. Lederer, notre école a pu, sans renoncer à sa vocation première qui est de former des interprètes el des traducteurs, creer puis developper un Centre de recherche en traductologie dont la qualité et la productivité ne sont plus à démontrer. Nous ne nous proposons pas ici de présenter en résumé les théories et les resultats auxquels ont about des annees de travail. nous renvoyons pour cela aux publications et dectorats du (iroupe de recherche en traductologie.

Nous tenterons par contre de rappeler, dans leur contexte historique, les motivations qui pousserent les responsables d'alors a aller de l'avant, nous insisterons sur la démarche méthodologique qui fut la leur et pour compléter cette approche qui vise essentiellement a retracer un parcours, nous indiquerons les horizons vers le'squels se poursuit aujourd hui notre chemin...

\section{CONTRE VENTS ET MARÉES}

Remontons en l'an 1.

En 1453, est crese lecole qui allail devenir quatre ans plus tard l'Ecole Superieure d'Interprètes el de Traducteurs (FSIT), grâce a l'intervention de M. Ciravier, professeur à la Sortome, qui a poné tout au long de sa carriere un intérêt constant aux problemes de la traduction. et notamment de la traduction théâtrale.

le premier objectif des interprètes de conférence qui y enseignent est de faire accepter l'idée que l'interprétatoon ne sauralt être enseigné par des professeurs de langue, pas plus que la chinurgie ne prourait l'être par un géneraliste qui $n$ 'a jamais tenu un sealpel. II faut confier cette tache a des praticiens contirmes.

l.urpence d'un approfondissement théorique se faisait néanmoins sentir. Il devait permettre de mieux comprendre la nature du proxessus interprétatif, d'ameliorer ba qualite de l'enseignement dispense, et d'atriver ainsi a une pratique plus exigeante el plus rassonné de l'interprétation consecuttve et simultanée. II s'apissait aussi d'ancrer l'enseignement de l'interprétation parmi les disciplines universitaires, ce qui sera chose faite en 1970, avec le rattachement de l'ESIT a I'Universile de Paris III.

Avant de siengager sur la voie de la réflexion theorique, Il fallatil voir quel était letat de lat recherche sur la traduction, en cette deuxième mottie des annees soixante. le tour d'horizon fut rapide

Premiere constatation : l'interprétation de conference n'a jamais véritablement fail lobjet doune étude. (ertes l: (ary. interprete de conférence a l'LiNESCO). avall con aacre un cours radiophomique en 1958 a linterprétation qui serat ensuite publié par M. Ballard dans Comme'nt fant-1/ rradure'? Quelques pages intedligentes, mais qui restaient encore it la surfice des choses.

Deuxième constatation : lev ouvrages war la traductoon étaient plus nombreux at plus consistants: Fedorov (195x). Malblanc (1961), Mounin (1963). Nida (19tht), Catford (1965), entre autres. La biblographie

\section{INTRODICTION}

1957-1994: I'ESIT a maintenant plus de 35 ans Sous la direction de M. (iravier, de D). Seleskovitch. 
internationale de la traduction publice par Babel, Revue intemationale de traduction. en $|\$ 6|$ recensait 670) titres. Mais. pour les pratıciens qui s intéressaient à la réflexion théorique, notamment D. Seleskovitch. C. Andronikof, A. Naudaud, John Coleman. la recherch: sur la traduction de l'époyue semblait $n$ 'avancer que dans les omières profondes creusees par la linguistique. en faisant fi de toute observation de la réalité et de l'experience.

Quatre pistes s ouvraient devant eux, comme autant de possihilités de fourvoyer la recherche en traductologie

1) Ia linguistique structuraliste, dans des approches aussi diverses que celles de Cassirer. Whorf ou Hjelmslev, avait remis a l'honneur un aspect de la philosophie du langage de Humboldt : le langage ne se contente pas de decouper la réalité perçue. il la détermine, imposant ainsi une réorganisation de lexperience sensible. De Malraux a Martinet. toute une génération fut ainsi convaincue de l'irréductible différence des civilisations et des structures linguistiques. Ce courant de pensée nous a laissé. en linguistique, des ouvrages passionnants d'un point de vue descriptif sur les particularites des langues amérindiennes. mais a embourbé pour des années la traduccolıgie dans une fausse problématique. achoppant sur des détails tels que: "Comment traduire neige dans les langues de pays subtropicaux ?"Nida, avec ses recherches sur la traduction biblique, a mainies fois soulevé le problème et Mounin lui a largement fait echo. Le "vide lexical" dans la langue darrivece est un obstacle aisément surmonlable, des lors que le traducteur ou l'interprète comprennent en situation le concept désigné par le terme dans le discours ou dans le texte.

Par contre. Nida a ouver une piste qu'il laisse très largement inexploré lorsqu' Il pose comme une évidence dans son ouvrage de 1969. The Theory and Practice of Translation:

Translating consists in reproducing in the receptor language the closest natural equivalent of the source language message', first in terms of neaning and se'condly in terms of style. (p. 12)

Cette phrase est caractéristique du stade apréscientifique" de la discipline: l'auteur introduit sans les définir. les notions de closest natural d'quivalent el de meaning.

2) Mais si l'on cherchait a approfondir la notion de meaning. on se heunail a l'époque a un autre courant de fond qui contrariatt et rendait illusoire toute avancée dans celte voie de recherche. La plupart des grands linguistes de cette époyue souffraient de ce que l'on appelle le «syndrome de la boîte noire". En s'interdisant toute definition interne du sens. de peur de sombrer dans un "mentalisme" non scientifique puisque non quantifiable. Hjelmslev, le pere de la glosermatique puis les distributionnalistes $\mathbf{B}$ /oomfield
Harris, Fries) permirent certes la encore à la linguistique descriptive de progresser. en mettant en evidence le réseau fonctionnel de dépendance des éléments linguistiques el leurs capacilés combinatoires. Mais ils entrainerent insensiblement la traduc. tologie dans le piege, alimente encore par la grammairc generative el transformationnelle de Chomsky. des structures profondes et les structures de surface de la langue. Le neferent n'est plus a l'honneur en tant que tertium comparatonis et pivot de l'ope. ration de traduction. (e courant donne nassance a des ouvrages comparationnistes comme celui de (atford ou celui de Malblanc et plus tard celui de Vinay et Darbelnet, ouvrages excellents pour qui étudie les langues, mass qui ne renseignatent guère sur le processus interprétatif

Les probièmes qu'ils évoxuent ne retiennent pas plus l'attention de $M$. Ciravier que de ceux qui gravitent à partir de 1974 autour de D. Seleskovitch. Directrice de recherches depuis que I'FSIT a obtenu I'habilitation a préparer des thèses de doctorat. L'interprète qui réexprime un discours au rythme de 1.50 mots/ minute ne passe pas par une analyse transformationnelle des structures des phrases prononcées, puisqu il ne les memorise pas en tant que telles.

3) Ce souci de scientifisme de ne saisir que le quantifiable avait encore été accentué par les progrès technologiques: l'informatique se developpait et l'on rêvail d'une "machine à traduire" qui, ouvrière infatigable, pourrait répondre aux besoins croissants de traduction. Mais ceux qui travallaient à sil mese au point furent bientôt tellement obnubilés par leur problé. matique specilique qu ils en vinrent a oublier la différence fondamentale qus existe entre l'homme et la machine... et finirent par croire qu'ils touchaient du doigt le coeur du processus de traduction alors qu'ils cherchalent des solutions a des problemes de programmation pour permettre à l'ordinaleur de surmonter la polysémic des mots et la polyvalence des phrases. Les psychologues vinrent leur prêter main-forte, à grand renfor de tests el d'expériences trop souvent arbitraires el coupés de tout contexte discursif. Paradoxalement, comme l'a souligne $D$. Seleskovitch en 1980, dans un article sur la machine à traduire (of. Seleskovitch 1984: 118-119). c'est l'impasise a laquelle ces premieres tentatives ont abouti qui a entraîné la lingusstique sur une voie nouvelle, celle des actes de langage, de la linguistique du texte et de l'analyse du discours, c'est-a-dire celle de la reconnaissance de lactivite langagière et du rôle des connaissances extra-linguistiques dans la compréhension.

Les recherches sur la machine a traduire et la psychologic cognitive ont sans aucun doute apporte des renseignements precieux, en particulier sur les aspects quantitatifs des langues, mais ne semblaient pas non plus pouvoir nourrir la réflexion de I'ESIT.

4) Le demier danger qui guettait la traductologie était indirectemenı lié a celui qui par ailleurs avait 
intuitivement le mieux compris la véritable nature du processus interprétatif et traductif: Edmond Cary. II dénonçait l "abstraction formelle" des constructions linguistiques et prox-lamait que la traduction était une operation sui generis. 1). Seleskovitch ne pouvall que souscrore a celte position. mais il ne fallait pas pour autant céder a la tentation de faire de la triduction un acte ansimilable a la création artistique, et it ac titre non thérorisable et non enseignable.

Peut-être est-ce précisement celle ampression d'être pris entre Charybde et Scylla, cette certitude que tout était a faire qui ont donne alux chercheurs de I'ESIT le courage de iengager sur une voic résolument nouvelle.

\section{LES FONISATIONS DE LA THÉORIE: INTERPRETATIVE : UNE METHODE EXPERIMENTALE. DANS UNF APPROCHE MULTIDISCIPIINAIRE.}

Tout était a faire et donc tout était à redéfinır, y compris le champ d'investigation lui-même el la méthode de recherche

Comme tout thercheur. D. Seleskovitch, qui fut la premiere a l'FSIT à se lancer dans la recherche theorique sur l'interpretation, aborde son sujet en posant un certain nombre d'hypotheses. Mais les siennes lut sont dictés par l'experience professionnelle

- I'interprete ne travaille pas sur la langue. mais sur le discours (elle ecrit pendant quelques annees ala parole", car il lui faudrat le temps de se dégager de lemprise salussurienne al préciser sa propre lemninologie (cf. Iaplace, a paraitre). Il faut donc cesser de vouloir élucider le processus de traduction en partant des caracteristiques de la langue:

- la langue, mise en axuvre dans le discours, ne fonctionne pas comme un corde et la communication ne se résume pas à une operation d'encodage el de décodage, à te qu il est convenu alujourd hui dappeler le amodele lélégraphique" (KerhratOrecchioni 1990): 25):

- La clef de voûte du proxessus interprétatif se situe dans la phase de compréhension, autrement dit dans la fameuse boîte noire, et c'est clle qu'il va falloir explorer, sans pour autani sombrer dans un mentalisme loufoxue !

Pour vérifier ou infirmer ces hypothèses, il fallait définir un champ expérimental et une méthocle.

l.e champ expérimental est tout trouves: c'est celui qu'oftre la pratique de l'interprétation de conférence. D. Seleskovitch aurait aimé pouvoir ne travailler que sur des situations "authentiques" : enregistrements de discours tenus en réunion el de leur interprétation in vivo par l'interprète. Matis dans ce cas, elle n'aurait pas eu la possibilité de comparer efficacement les prestations des interpretes et d en tirer des conclusions généralisables, puisque, dans la pratique, il n'est pas donne à $x$ interpretes d'imerpréter un mëme discours vers une même langue dans des conditions identiques.
Flle choisit donc de crér la serre d'experiences similaires dont elle avait hesoin pour amiver a des résultats significatifis en plaçant des interprètes de contérence chevronnes dans des conditions de travail auss semblables que possibles à celles de la vie profession. nelle (l’interpritte sait quel est le sujet qui va être aborde, a connait les fonctions ou pevitions particu lieres de loraleur, il n'entend qu'une seule fois le discours. ek.). Fille s'interdit donc de travailler sur des exemples de traduction crés ad hes, pour etayer une démonstration, et nacepte le montage dexpe rences qu'a condition que celles-ci solent auss proches de la realité que powsible el qu elles a fassent toujours avec des interpretes chevronnes. car seule la prestation globalement réussie peut renseigner sur la réalité du processus interprétatif. Flle réunit son premier corpus, celul yui servira de base a langues. langage et miniorier : un méme discours. interprété en consecutive, apres mise en stfuation. par plusieurs collegues, avec enregistrement et retranscription des interprétations et observation des notes prises par les differents interprètes?

Lil démarche peut paraître evidente à tous ceux qui font aujourd hui de lat recherche en traductologie. A l'époxque, elle était révolutionnatire. Depuis, lout Iravall sérieux sur lo sujet $x$ base sur des corpus comparables. L iouvrage de refierence de $M$. Iederer sur la traduction simultance $(1981)$ en est un des meilleurs exemples, mais l'on pourrait également citer, pour la recherche en interprétation, Dejean le Feal, Ixonovan. ctc.

("est le princepe de l'exploitation methodicyue d'un corpus yui a permis a l'ESIT de mettre le doigt sur quelques-uns des principes fondamentaux de la thécice interprétative. Ainsi. l'analyse méticuleuse at synchronisés des enregistrements diune seance du

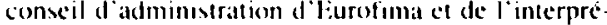
tation des débals par deux interpretes (voir lederer 1981. pour le montage exact de l'expérience) a permis a M. lederer :

- de démontrer yue la compréhension du vouloir dire de l'oratteur par l'interprète, préalable indispensable at sa réexpression dans une autre langue, se fait par "unites de sens" qui ne coincident pas nécessairement avec des unites semantiques ou syntaxiques. comme l'a longlemps presuppose la recherche en linguistique :

- de faire ressortir le rôle du contexte cognitif el des complements cognitifs dans ce processus de comprébension, en insistant sur les phénomènes d'anticipation sémantique et cognitive et sur les différentes fonctoons de la mémoire:

- d'aboutir à une conception du rapport entre langue, langage et pensece.

Si lobservation des donnés expérimentales a permis d'aboutir a l"elatoration de ces theses. cest grâce aussi - me semble-1-il - à l'independance des chercheurs vis-à-vis de tout dogmatısme. Nul n'echappe certes el surtoul pas ceux qui sien défendent - à 
la grille interpretative d'une Weltanschauung. Mais encore faut-il nuancer. Ainsi un chercheur en traductologie comme Otto Kade était-il ienu de passer ses données experimentales au crible d'une grille particulièrement contraignante (cf. Laplace 1991). Les traductologues qui s'inspiraient de la linguistique structuraliste ou distributionnaliste étaient, cux, hantés par un anti-mentalisme dont Searle $(1992: 13)$ explique - sans l'excuser - l'origine

. ne have a terror of falling into Cartestan dual. ism. The hankrupicy of the Cartesian tradition and the ahsurdety of supposing that there are on's kinds of substances or properties in the n'orld. "mental" and aphysical" is so threatening or us and has such a sordid history that we are' reluctan wo concede anything that might smack of Carte. sianism /... / Any sort of mentalism that recog. nizes the obvious facts of our a distence is regarded as automatic ally suspers.

Seleskovitch et Lederer, ainsi que tous ceux yui ont par la suite travaillé avec elles, n'étaient pas entravés dans leur réflexion par ce tabou et acceptaient de reconnaitre le rôle de la conscience du sujet. là wù il se manifestait, souscrivant bien avant I'heure à celte phrase de Searle

...the mental state of consciousness is just an ordinary bological. that is, physical. feature of the hrain

Elles ont cherché. auprès de specialistes d'autres disciplines, une confimation des deductions qu'elles avaient tirées des données expérimentales. Cette ouverture transdisciplinaire a pris deux formes:

- celle d'un simple enrichissement intellectuel. d'une source de références, lorsqu'il s'agissait des travaux de Plaget, ou de ceux de E. Coseriu sur le langage ${ }^{3}$ :

- ou celle d'une véritable coopération en matière de recherche lorsque $D$. Seleskovitch et $M$. Lederer travaillèrent en équipe avec $M$. Pergnier en tant que specialiste du fonctwonnement de la langue et J. Barbizet. specialiste des mécanismes céebraux au sein du (i.F.L. (Ciroupe d'étude du langage) de I'Universite de Paris XII et obtunrent ainsi une confirmation neuro-psychologique des thèses avancées par I'ESIT

Dans tous ses travaux de recherche, l'ESIT s'est fixé également un autre principe de base. celuı du "parter clair". Tout interprète de conférence a eu l'occasion d'apprécier la différence qui existe enıre une expression precise et nuancé et le recours systematique a un jargon impénétrable. Il est certes indeniable que tout approfondissement de la reflexion dans un domaine donné entraine la création d'un certain nombre de termes ad hoc, pour rendre compte de notions nouvelles. Mais un peu de vigilance empêcherait souvent la prolifération de ces néologismes qui hérissent les textes de certaines publications actuelles, sans que l'on perde pour autant en rigueur analytique.

\section{LA CREATION DU CENTRE DE \\ RFCHERCHE : L'ESSOR DE I.A THEORIE INTERPRETATIVE.}

Une recherche d'envergure en traductologie ne pouvaıt être l'affaire d'une seule personne. Aussi, trè̀: tôt, $M$. Lederer vint rejoindre l'ESIT et lui apponer ses compétences d'universitaire, d'interprète et de chercheur. De sa collaboration avec D. Seleskovitch résultèrent quelques-uns des grands ouvrages de réflexion sur l'interpretation et la traduction. et la constitution d'une «thécoric interprétative". Mais pour pouvoir a la fois elargir et approfondir les recherches et assurer la perennite de l'ESIT en tant qu'institut universitaire en etoffant le corps des enseignants en poste, il fallait

- trouver parmi les professionnels qui enseignaient l'interprétation ou la traduction un certain nombre de personnes suffisamment motivées par l'intérêt d'une recherche theorique. pour dégager le temps nécessaire a la rédaction de thèses de doctorat : ce furent le cas en interpretation de C. Thiery. qui elail ('hef du Service de l'interprétation au ministere des Affaire. étrangères, de K. Dejean Le Féal, de C. Donovan, aujourd hui Directeur de la section Interpretation et de moi-même et en traduction de $C$. Durieux. F. Herbulor, E. Drozdale :

- faire venir a I'ESIT des universitaires qui pouvaient lui apporter une precieuse contribution (ce fut le cas, entre autres, du professeur F. Israèl, spécialiste de la traduction de Shakespeare el actuellement directeur de la section Traduction):

- crér une veritable structure de recherche propre a I'ESIT. C"est ainsi qu'en 1976 fut crés le Centre de recherche en Iraductologie. Cela a permis a I'ESIT d'élargir son champ d'investigation, puisque l'experimentation peut désormais se faire à partir d'un nombre appreciable de langues, en fonclion des compétences propres aux chercheurs qui rejoignent lo centre. et sur des supports qui vont des conférences internationales à la puesie, en passant par les textes publicitaires. Ce champ s'est encore clargi avec lintroduction d'une MST d'interprétation en langue des signes ${ }^{4}$ qui donne à la section Recherche la possibilité d'érudier les rapports entre langue audio-vocale et langue gestuelle el de vérifier que la theoric interpretative de la traduction $s^{\circ} y$ applique, comme à toute autre paire de langues. Une these de doctorat a ete soutenue le 21 octobre 1994 par Ph. Séro-Cuillaume sur le sujet.

La section Recherche prépare à un Diplôme d'études approfondies (D.E.A.) et à un doctorat de traductologie. Pour préserver le lien entre pratique professionnelle el actovite universitaire, lien qui fail l'un des atouts de l'FSIT, la section Reclierche 
n'admet en D.E.A. que les étudiants qui ont. outre les diplômes requis et un projet de recherche, une pralique professionnelle dau moins trois ans. Chaque année I'ESIT inscrit une quin/aine d'étudiants en séminaire dectoral.

Le centre de recherche et la formation doctorale ont largement coneribué a assurer la diffusion de la theorie interpretative de la traduction dans le monde. grâce aux nombreux chercheurs etrangers yul ont fatt un doctorat a I'ESIT. Leurs travaux ont permis de prouver que cette thérie, né de l'ctude de l'interprétation de conference, conservait toute sa pertinence lorsqu elle étalt appliquéce au domaine de la traduction Ecrite. Il est impossible de faire iel une liste exhaustive, citons simplement J. Delisle, professeur à I'Universite d'Ollawa, et M. Cormier. professeur it l'Université de Montréal, Jung Wha Choi, professeur a l'Université Hankuk en Corée. He Ping Zhao, interprète et traducteur chinois, et bien sur nos collegues, A. Hurtado, G. Misri, C. Driesen. th bien d autres.

la recherche théorique a eu des retombers didace. tiques qui n'ont pas remis en cause fondamentalement les options adoptees par les enseignants-interpretes ou - Iraducteurs sur la base de leur expérience pratique. mais ont permis par contre de justifier les grandes orientations et d'améliorer certaines pratiques. Les enscignants en interprestation disposent aujourd hui

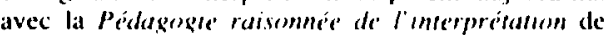
D. Seleskovitch et M. Lederer -- dont la traduction anglaise va sortur prochainement aux Etats-l/nis sous le titre A Sisvematic Approsach of Teaching Interporeration - d'un ouvrage de référence qui trate de façon approfondie de lenseignement de la consécu. tive (prise de noles et progression comprise) et de celui de la simultanéc, en prenant position sur le délicat sujet du travail vers le $A$ ou vers le $B$ et en proposant toute une sére dexercices préparatoires à la sumultantece. Ce travail, commandite par la Commission de la Communaute europeenne en 1984, contribuc a la diffusion des proncipes didactiques de l'ESSTT dans le monde. tout particulièrement lorsqu il sagit de créer dans des pays en voice de développetment des centres de formation a leinterprétation et a la traduction, travail de longue haleine, prepare pendant des annees par K. Dejean l.e Fiéal, en Firềme-( )rient.

Les enseignants en traduction disposent d'un ouvrage similaire pour la traduction technique:

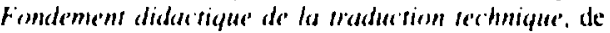
C. Durieux.

Tous ces travaux nous ont bien entendu amenés a améliorer encore notre systène de sélection a l'entréce dans notre école : en traduction, une étude a été réalisée en 1991 sur les aptitudes et les prérequis nécessaires aux apprentis traducteurs. En interprétation. nos

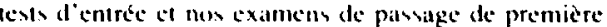
en deuxieme annece nosus permellent deshernir un taux de réussite au diplome plus gatssaisant, tout en maintenant le niveau qui nous a valu notre reconnasssance internationale

Matis le travanl n'est pas pour autant achevé. Nous avions lance, par exemple, un proget de recherche sur la traduction à vue. Celte étude visait à déterminer comment sopere la compréhension du sens à partir de la perception visuelle du texte et elle avait été amorcée en collaboration avec la section d'éludes el fabrications den télécommunications (SEFT) du ministère de la Défense. In legiciel fut conçu et testé pour permettre de suivre, grâce à des systèmes de visée très perfectionnes, le déplacement du regard sur le texte. Mais a ce jour. ke matéricl. pesurant beaucoup plus performant que celui utilise habituellement par les instituts de marketing pour suivre le parcours du regard d'un consommateur sur un mailing. n'a pas encore atteint le degré de précision nécessaire. métier...

II nous faudra donc remelure notre ouvrage sur le

\section{I.ES PERSPECTIVES : PHILOSOPHES, LINGIUISTES ET TRADUCTOLOGUES : I.A MARCHE: F.N AVANT}

Depuis lit fin des annees soixante, les chercheurs de l'ESIT ont travaillé avec beaucoup d'opiniâtreté pour faire de la traductologie autre chose que l'étude comparative de deux proxluits achevés et considérés comme sémantiquement équivalents. bien que rédigés en deux langues differentes. Ils ont imperturbablement mantenu leur cap, envers et contre loutes les modes. el poursuivi leur analyse de la traduction comme un double processus de compréhension d'un sens lqui grace au hagage cognitif va au-dela du seul semantorme des motsl et de réexpession de ce sens en recourant à un autre instrument linguistique. l.e discours stait ainsi ćtudié comme un acte de langage. par lequel un locuteur exprime son vouloir-dire, en tenant comple des conditions spécitiques de la situation de communication et de l'effet prexluil par le discours sur l'auditore, en modulant par exemple spontanémem le rappont entre l'implicite et l'explicite

("eut donc avec traucoup d'interêt que I'ESIT constate aujourd hut que linguistes et philosophes travaillent de plus en plus sur les aéchanges langagiers". l' «interaction verbale". "l'interlocution". Le chemin parcouru a été long: il a fallu la théorie des acter de langagee, lanalyse du discours en la grammaire textuclle pour que, peu à peu. el non sans rélicences. les linguistes acceptent de réintegrer dans leur étude le contexle et l'action des interlocuteurs comme indıvidus doues d'une intentionnalite. Searle (1964-1972), au déhut de sa théorie des acter de langage, se defendait encore de trivailler sur autre chose yue la langues. Ia l'analyse du discours n'a longlemps travaillé que sur la mise en acurre de la langue dans la parole.

Mais peu à peu les choses évoluent. Les linguistes yui n'aimalent jadis rien tant que travailler sur des exemples fatriqués de manière ad hes pour les besomns de lat cause, changent de méthoxk et Kerbrat-Orecchioni (16x) : 4.5.46) souligne que l'on assise depuis quelques annees it un deplacement méthodologique important : alors que "dans les annees soixante. $\mid . .$. le rejet des corpus. le mépris des performances effectives. se 
voyaient même justifies theoriquement". on constate aujourd 'hui "une rehabilitation de l'empirisme descriptif". ce qui implique ale souci de travailler a partir de corpus enregistrés et soigneusement retranscrits".

Il est egalement réconfortant de lire dans le dernier ouvrage de Searle :

In my ven the philosophy of language is a branch of the philosophy of mind; therefore no theory of language' is complete without an accesum of the relations betwe'en mond and language and of how meaning - the derised intentionalipy of linkuistuc elements-is grounded in the more balogicalls basic intrinsu intemionaliny of the mind / bruints.

et de voir qu'il veut en finir avec les sempiternelles theories sur l'encodage et le décodage, basées selon lui sur une "conception prédarwinienne" pour s "interesser enfin au fonctionnement du cerveau humain

I nats to pal the final nail in the coffin of the theory that the mind is a computer program.

I..) He trit to find out how humans might ressm. ble our computational models rather than tring to figare out how the conscious human nuind acritully norks?

Et c'est avec bonheur que nous lisons sous la plume de Kerbrat-Orecchioni d'intéressantes études sur les anticipations. les rétro-actions, l'implicite. et surtout que nous trouvons soudain une reconnaissance pleine et entière du travail interprétatif du récepteur dans l'acte de communication :

Il serait absurde de nier l'idée que préexiste aux echanges communicatifis un ensemble de règles linguistiques relativement stables. Mais elles sonı floues, variables. dépendantes du contexte : elles ne sauraient donc a elles seules donner la clé du sens des énoncés actualises. Sens que le récepteur ne se contente pas d'extraire de l'enveloppe signifiante où il se trouverait sagement enclos. mais qu'il doit (rekonstruire au lerme d'un acalcul interprétatif " complexe ${ }^{\mathrm{x}}$.

Quant à l'engouement pour l'intelligence artificielle, il semble avoir aussi fait son temps, ou du moins avoir été ramené dans des limites plus raisonnables, si l'on en croit J. Ninio, biologiste et Directeur de recherche au CNRS qui écril dans L'mpreinte des sens. : Percoption. Mémoire. Langage' :

...l'intelligence artificielle $[. .$.$] néglige le fait que$ dans la pratique le discours suppose une foule de connaissances implicites, de telle sorte qu'on peut faire passer un message en disant le contraire. Une phrase peut dire exactement le contraire de ce que. grammaticalement, elle est censé dire. [... Mais surtout l'intelligence artificielle oublic que chez l'homme, les mots s'adressent directement au psychisme. Quelques paroles d"apparence anodine peuvent plonger un individu dans won passe. eveiller en lui des ventinkents de remords. de peur, de pitié. de tendresse, ou encore le mettre hors de lui. Nulle conversation n'est innocente.

C'est donc avec beaucoup d'optimisme que I'ESIT envisage l'avenir: si les principaux courants de la recherche sont désormais d'accord sur les notions les plus fondamentales. I'ennchissement mutuel d'une discipline a l'autre devient possible. Ft c'est ainsi que nous esperons donner vic dans les années venir à l'école doctorale Sicien'es du langage' Arts du spertacle', (immanicarson yui s'est constituee a Paris III el qui regroupe sept D.E.A. : sciences du langage, phonétique, didactologie des langues et des cultures. philosphie du langage et de la communication, theâtre et arts du spectacle. recherches cinematographicues et audiovisuelles. et traductologie. Cet ensemble pluridisciplinaire, composé de filières transversales. et placé actuellement sous la direction de $M$. Lederer, perme1 aux chercheurs de I'ISSIT d'avoir avec leurs collegues de fructueux contacts et seminaires. ("est ainsi par exemple que les sémınaires de Francis Jacques (1979, 1982, 1985), specialiste inconteste de l'approche philosophique du dialogue. nous apportent une précieuse ouverture sur une problématique plus vaste.

\section{CONCLUSION}

Personne ne saurait nier yue l'ESIT a reçu une impulsion décisive grace à la forte personnalité de D. Seleskovitch. Praticıenne émérite. elle a entrepris de réflechir sur son métier, proussée a la fois par une curıosite théorique el par le souci pedagogique de transmettre son experience pratique. Fille a mené cette reffexion avec une grande indépendance d'esprit, dans une epoxpue ou triomphait une sortc de platonisme inavoue. Elle a su ramener la traductologie dans les voies d'un sage aristotélisme pour lequel il n'est pas de connaissance sans une confrontation permanente de l'idée et du sensible, unis dans une tension dialectique. Ei elle n'a jamais cessé de considérer comme primordiale la iransmission de l'experience, c'est. a-dire la pédagogie de l'interprétation et de la traduction. n'hésilant pas a s'attacher a des aspects pratiques trop souvent négliges par le théoricien. mais pourtant essenticls pour les futurs interpretes et traducteurs.

L'ESIT est-elle encore aujourd hui animes par la dynamique de cette impulsion première? A-1-elle su éviter le ronron qui accompagne trop souvent le processus de l'institutionnalisation? Nous sommes, il est vrai, a la fois juge et partic.

Il nous semble cependant que nous n'offensons pas l'objectivité en constatant que l'ESIT poursuit sans dogmatisme l'ceuvre entreprise. Nous n'ajouterons que deux exemples. à ceux déjà éroqués precedemment.

- M. Lederer vient de publier un ouvrage sur la traduction ${ }^{9}$ dans lequel elle en examine de manizre approfondie les aspects théoriques et pratiques, en se 
réferant constamment à des exemples tores de l'expérience.

- Les travaux de recherche entrepris en collabx) ration avee une équipe de neurologic avaient été sus pendus par la mort de J. Barhizes. Ils vont être repris.

En outre, une douraine de theses de doctorat sont en cours d'achevement ou sur le point d'être soutenues, sur des sujets aussi divers que la traduction litteraire chinois-français, la traduction des titres, lat traduction des jeux de mots. le sous-titrage. la traduction juridicue.

pour fimr, nous voudrions souligner un aspect non négligeable de notre activité dome l'actualité ne saurait etre méconnue. Par la qualite de onn enscignement. soutenu constamment par un efforl de reflexion théorique, I'ESIT a su se faire reconnaitre comme institul universitaire. Personne ne nera le prestige qui en a rejailli sur les protessions d'interpretce el de traducteur, III le surcroil de renommet intemationale qui en resulte pour l'ecole.

A l'heure où l'universite s'interroge sur se's propres finalites et sur les conditions de transmission du savoir. les enseignants et chercheurs de l'ESI'T souhatent continuer a contribuer, avec tous les chercheurs interesses, a cetle confrontation permanente des visés thériques el pratiques, sitns perdre de vue leur finalite propre: former des praticiens competents.

\section{COLETTE LAPLACE Viniersite de la Sorbonne Nouvello Parris III. ESTT. Paris. Framer}

\section{Notes}

I. Nous reprenons ici la terminologie de M. Pergnier qui réserve le terme d'ambiguîte a une situation d'interlexution, puisquelle suppose la avolonte de transmettre une information univopue d unc conscience à une autre conscience"» et préfere parler de "polyvalence" pour des faits de langue.

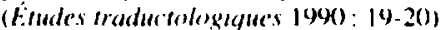

2. Fille prend alors conscience que le terrain n est pas préparé pour assurer la réception d'un tel travail el décide de commencer par rédiger un premier ouvrage, l'Interprife' desms le's conférences inter'nafonsles. (1968). afin d'expliquer préalahlement sans tenter encore de démontrer par l'analyse" d'un corpus ce quest pour elle le travail de l'interprète.

3. Coseriu a slabore une conception du langage d une extrême cohérence qui se rattache a la penséc aristotélicienne et humboldtienne el propose en particulier une approche de la competence (ćlocutoire, idiomatique et expressive) domi les avanceses nont pas encore tete entierement assimileses par la linguistique actuelle

4. Laction a été mené en collaboration avec le Centre d'études et de formation pour l'enfance inadaptée (CNI:FE:L)
5. ")( ) pourralt encore croire que mon point de vae represente simplement. au sens que Saussure a donné a ces termes. une etude de la "parole" plutiot yue de la "langue". Je prétends cependant qu'une ćtude appropriéc des actes de langage est unc slude de la langue." (Starle 1972: 53.54)

6. John R. Searle, The Redesecrers of the Mind I092, p. XI

7. IhI, pp. XI 247

8. ('. Kerbrat-Orecchioni, Le's interdations serbales. 19(0), p. 2K. On peut loutefois $x$ demander si $k$ dépassement de la position antérieure est bien total. lorsqu'on retrouve sous sa plume les termes de canal ou de cokte qui rappellent dangereusement la conception télégraphique des annés soixante: "La communication est multicanale el pluricodique". p. 47

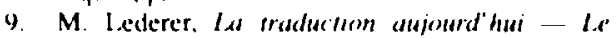
modele imeroméfonff. Paris, Hachetle, 1904

\section{RFFERENCES}

CARY, Edmond (1985): Comment faut-1) raduire" (coum de 1958). I.ille. Presses Universitaires de l.ille. $94 \mathrm{p}$

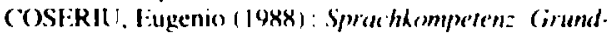
iege der Theorre des Sprechens. Tübingen. Francke Verlag, XIII, $299 \mathrm{p}$.

DELISISL.E. Jean (1984) : l'anulyst' du discours comme methrode de traductum. The'orre ef prarique" Otawa. Editions de l'Oniversite d'Ottawa. $282 \mathrm{p}$.

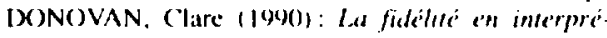
tartom, these de dextorat, dactylographiec, Paris, $50.3 \mathrm{p}$.

DURIE:UX. Chrostine (198X): Fondeme'mt dedactichu de la raduction terhmique, Parts, Didicr Frudition. $171 \mathrm{p}$

JACQUJES, Francis (1485): l'espace loglame de limer/oxurion. Paris, PUF, 640 p.

KERBRAT-OREC(CHIONI, Catherine (1986) : L'impli. rlle. Paris. Armand Colin. $404 \mathrm{p}$.

KERBRAT-ORE('CHIONI. (atherine (1990): Le's inseractums rerbale's, Paris, Armand colin, wome 1. $318 \mathrm{p}$.

1.API.ACE, (olettc (à paraître): Théorie de langage et theorie de la traduction las concepts-clefs de

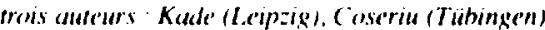
Seleskoniteh (Paris), these de doctorat, dactylographies, Paris III. 1991. 4.59 p. la paraitse che? Didier, debut 1605)

IEDERE:R. Marianne (1981): La traduction simal ame'e Fonderments the'ernque's. Paris, Minard lettres Merlernes, $454 \mathrm{p}$.

I.EDERER, Marianne et Danica SEI.ESKOVITCH $(1984)$ : Interpresere pour tradure, Paris, Didier Erudition. $312 \mathrm{p}$

LEDERER, Marianne (dir) (1090): Euctes roduc wologique's, en hommage a D. Seleskovitch, Paris. Minard lettres Mexdernes, $286 \mathrm{p}$.

L.FDFRFR, Marianne (199.4) : La traducthom aujourd hu Le' modele interpréfont, Paris, Hachette. 
LYONS, John (1989): Introduction to Theorefical Linguistics, Cambridge, Cambridge University Press, X. 519 p

MOUNIN, Georges (1963) : Les prohldmes théoriques de la traducrion, Paris, Gallimard, $309 \mathrm{p}$.

NIDA. Eugene A. (1964): Toward a Science of Translating. Leiden. E. J. Brill. X. 331 p.

NIDA Eugene $A$. and C. R. TABER (1982): The Theory and Practice of Translarion. Leaden, E. J. Brill, VIII, 2 I $\mathrm{p}$

NIVIO, Jacuues (1991): L'empreinte des sens: Perception. Memoire. Languge. Fditions Odile Jacob. $310 \mathrm{p}$.

SEARLE, John R. (1992): The Rediscovery of the Mind, The MIT Press, Cambridge. Massachuselts, London, England, 285 p.

SELESKOVITCH. Danica (1968): L'interprete dans les conferences internationales, problemes de langage ef de communication. Paris. Minard Lettres Modemes, $257 \mathrm{p}$.

SELESKOVITCH, Danica (1975): Langage', langues et mémoire, clude de la prise de nofe's en interprifation consécwlive. Paris. Minard Lettres Modemes, 277 p.

SEL.ESKOVITCH. Danica et Marianne LEDERER (1984): Interprete't pour traduire. Paris. Didier Erudition, 312 p.

SEI.ESKOVITCH. Danica et Marıanne LEDERER (1989): Pédagogic raisimné de linterprétanion. Paris. Didier Erudition, $312 \mathrm{p}$. 